\section{P-165 THE DEVELOPMENT OF A MULTIDISCIPLINARY PALLIATIVE CARE OUTPATIENT CLINIC}

Anne Goggin, Kerry Worsfold, Fran Walton. Countess Mountbatten House, Southampton, UK

\subsection{6/bmjspcare-2018-hospiceabs.190}

Aim To develop a model of Palliative Care Outpatients that allows the patient access to appropriate professionals for their needs in a single visit.

Background There is good evidence that optimal palliative care of patients and their families is delivered through multidisciplinary teams. In 2015, to offer a more flexible and efficient service to our patients, we set up parallel medical and nursing outpatient clinics at the hospice. Our experience in running the outpatients and the identification of a need for therapy input led us to see the value of multidisciplinary consultations. We gained a place on our Trust's 'Team QI Fellowship Programme' which gave us a valuable framework for this new development.

Project design Adoption of the PDSA cycle (Plan, Do, Study, Act) model. Creation of a Driver Diagram to establish our overall goal and the steps we needed to complete to achieve the goal. Process mapping of current outpatient service. Development of a patient questionnaire. Feedback from staff delivering outpatients.

Findings

- Administration process lacked efficiency

- Admin team required further training

- Identified that additional admin resource needed to deliver the service efficiently

- Outpatient environment felt isolated

- Feedback from patients: outpatients is an acceptable and helpful environment for accessing specialist palliative care.

Actions Consultation with staff and volunteers to make changes to allow the MDT clinic to run alongside Drop-In service, creating a more welcoming environment for patients and carers. Accessed training for the admin team on the booking system and streamlined booking processes. Development of a business case for additional admin resource for outpatients.

Outcome We launched our new MDT clinic in February 2018. Results To date we have found our MDT clinic to be an effective way of delivering outpatient assessments.

Conclusion The patients have received a more holistic service in a single visit.

\section{P-166 VOLUNTEER HOME VISITING SERVICE}

Danielle Smeeton. LOROS Hospice, Leicester, UK

\subsection{6/bmjspcare-2018-hospiceabs.191}

Background Previously known as the Family Support Service, paid staff visited patients in their own homes to provide companionship, reduce social isolation and offer respite/a break to carers and families. This service was reviewed in 2013 and it was felt that although patients and carers found the service to be very beneficial, we remodelled the service so that it was less costly and could be offered to more patients, living at home. In 2014, the Home Visiting Service was launched as the remodelled Family Support Service. The Home Visiting Service provides volunteers to support patients and families in their own homes and the community.
Aims To give patients company, conversation and support in their own homes; and also the opportunity to go out and engage in community activities. The service can also benefit families and carers, to give them a break from their caring role, with peace of mind that their loved one is safe and occupied.

Methods High calibre volunteers are recruited and trained to visit patients in their own homes either weekly or fortnightly, for up to four hours (including travel). The volunteers can support patients by providing company and conversation but also assist with daily living, enabling patients to continue with their hobbies and can take patients out into the community.

Results 80 patients are currently being visited by 60 volunteers. Feedback from patients and families is very positive and some patients have said that the service is 'invaluable' and 'life altering'. The service has benefitted patients in the following ways: Reduced patients' social isolation; Enabled patients to play games/do quizzes/crosswords; Enabled patients to continue with hobbies; Enabled patients to get to appointments; Enabled patients to go out into the community to undertake physical exercise; Enabled patients, carers and families to have a break.

\section{P-167 TELEHEALTH PILOT: DEVELOPMENT AND SETTING UP OF A TELEHEALTH SERVICE FOR PALLIATIVE CARE PATIENTS}

1,2Emma Randall. 'Weldmar Hospicecare, Dorchester, UK; ${ }^{2}$ Macmillan, South West, UK

\subsection{6/bmjspcare-2018-hospiceabs.192}

Weldmar Hospicecare has growing numbers of patients being referred to the service who want to be supported in their own homes for as long as possible. Weldmar are working in partnership with Macmillan who are providing funding for an 18 month secondment post to set up and lead a telehealth pilot. Therefore enhancing the service offered to Weldmar patients and giving clinicians another tool to support and monitor patients. Telehealth is already being used in the UK for patients with long term conditions such as COPD and heart failure but there are limited numbers of palliative care patients using a telehealth service.

The pilot consists of a POD which has a protocol uploaded to it that has been written using recognised palliative care outcome measures; these are the Barthel Index and the IPOS scoring system. Patients $\log$ on daily completing the pre-set questions - this will monitor changes in symptoms and also level of need. The results are stored on the POD so clinicians visiting the home can access these, or have the option to access them remotely from their PC to review the data the patient has inputted.

The PODs have the capability for video-conferencing and allow clinicians to use this to discuss with patients the data they have inputted and also carry out assessments of patients face to face via Skype. Patients have embraced the pilot and use of the POD. They report the POD is quick and easy to use, they like that clinicians can monitor them remotely. Clinicians are adapting to having another tool to use to support patients and having access to a wealth of data about patients. Also having the ability to monitor changes in symptoms without being reliant on patients relaying to them how they have been over a set period of time. 